Fisioter Bras 2021;22(6);791-808

doi: $\underline{10.33233 / \mathrm{fb} . v 22 i 6.4919}$

\title{
ARTIGO ORIGINAL \\ Caracterização da força e da função muscular nas disferlinopatias em amostra brasileira
}

Characterization of muscle strength and function in dysferlinopathy in a Brazilian sample

Isabela Pessa Anequini, M.Sc. ${ }^{*}$, Jéssica Marim de Lima, M.Sc. ${ }^{*}$, Silvana Amanda do Carmo $^{* *}$, Juliana Aparecida Rhein Telles, M.Sc. ${ }^{* *}$, Mariana Callil Voos, D.Sc. ${ }^{* * *}$, Fátima Aparecida Caromano, D.Sc. ${ }^{* \star \star *}$

*Universidade de São Paulo (MRCUSP), LaFi.Com, São Paulo/SP, " Doutoranda em Ciências da Reabilitação pela Faculdade de Medicina da Universidade de São Paulo, São Paulo/SP, ${ }^{* * *}$ Pesquisadora do Curso de Fisioterapia da FMUSP, Professora da Universidade Ibirapuera e PUC/SP, São Paulo/SP, ${ }^{* * * *}$ Profa. do Curso de Fisioterapia MRCUSP, Orientadora no Programa de Pós-Graduação em Ciências da Reabilitação da Faculdade de Medicina da Universidade de São Paulo (FMUSP), LaFi.Com, São Paulo/SP

Recebido em 12 de setembro de 2021; aceito em 4 de dezembro de 2021.

Correspondência: Prof ${ }^{a}$. Dra ${ }^{\mathrm{a}}$. Fátima Aparecida Caromano, Universidade de São Paulo (USP), Rua Cipotânea, 51, Cidade Butantã 05360-000 São Paulo, SP, Brasil

\footnotetext{
Isabela Pessa Anequini: isa.anequini@gmail.com Jéssica Marim de Lima: jessica.marim1@gmail.com Silvana Amanda do Carmo: carmo.amanda@gmail.com Juliana Aparecida Rhein Telles: juliana.rhein@usp.br Mariana Callil Voos: ftmarivoos@gmail.com Fátima Aparecida Caromano: caromano@usp.br
}

\section{Resumo}

Estudo de caracterização da força e da função muscular nas disferlinopatias para estabelecer biomarcadores de habilidades motoras com amostra de 40 pacientes, tendo sido avaliados força muscular (Medical Research Council - MRC), percentual de MRC, tempo de execução para deambular e escores nas Escalas de Vignos, Egen Klassifikation, Avaliação Funcional para Distrofia Muscular de Duchenne (FES-DMD) e 
North Star Ambulatory Assessment adaptada. Prevalência da disferlinopatia de 25,5\% na amostra total de distrofias (1340), idade média de 36,5 anos, 52,5\% do sexo masculino e 75\% deambuladores. Músculos mais fracos: abdominal, glúteos, íliopsoas, isquiotibial, quadríceps femoral, tibial anterior e deltoide médio. Correlação forte entre MRC e tempo para deambular $(r=0,77)$ e, muito forte da MRC distal de membros inferiores com aNSAA $(r=0,90)$. Interação da MRC dos membros superiores e inferiores nos segmentos proximal e distal $(p<0,001)$, sendo mais evidente em membros superiores que inferiores. Taxa variável de progressão da doença com $60 \%$ dos pacientes moderadamente ou gravemente afetados, com mais de 12 anos de doença. Estudo mostra que padrão de fraqueza muscular dos brasileiros com disferlinopatia é proximal e distal dos MMII, com comprometimento associado da região proximal dos MMSS, além de elucidar as habilidades motoras em relação ao processo de locomoção e disfunções cardiorrespiratórias.

Palavras-chave: distrofia muscular do cíngulo dos membros; doenças neuromusculares; disferlina; debilidade muscular; destreza motora; avaliação da deficiência.

\section{Abstract}

The study of the characterization of muscle strength and function in dysferlinopathy to establish biomarkers of motor skills. Sample of 40 patients with muscle strength with MRC (Medical Research Council), MRC percentage, timed motor performances for walking, Vignos scales, Egen Klassifikation, Functional Assessment for Duchenne muscular dystrophy (FES-DMD) and North Star Ambulatory Assessment Adapted (aNSAA). Prevalence of dysferlinopathy of $25.5 \%$ in all dysthophies studied (1340), mean age of 36.5 years, $52.5 \%$ of males and $75 \%$ of walking. Weak muscles: abdominal, gluteus, iliopsoas, hamstrings, quadriceps femoris, tibialis anterior and medial deltoid. Strong correlation between MRC and time to walk (mean $r=0.77$ ) and very strong MRC distal lower limbs with aNSAA $(r=0.90)$. Interaction of MRC of upper limbs and lower limbs between the proximal and distal segments $(p<0.001)$, being more evident in upper limbs than in lower limbs. Variable rate of disease progression, with $60 \%$ of patients moderately or severely affected with $>12$ years of disease. The findings characterize the pattern of muscular weakness of Brazilians with dysferlinopathy as proximal and distal lower limbs, with associated involvement of the proximal upper limbs, besides elucidating the motor abilities in relation to the locomotion process and cardiorespiratory diseases.

Keywords: muscular dystrophies limb-girdle; neuromuscular diseases; dysferlin; muscle weakness; motor skills; disability evaluation. 


\section{Introdução}

As disferlinopatias formam grupo de doenças neuromusculares genéticas, de herança autossômica recessiva [1,2], com alterações na produção da proteína disferlina, presente na membrana das fibras musculares e, que é codificada a partir do gene DYSF, localizado no cromossomo 2p12-14 [3,4]. Os principais fenótipos encontrados são a miopatias de Miyoshi (MM) e a distrofia muscular de cinturas (DMC) do tipo 2B (DMC2B) [2].

A DMC2B é a segunda forma em frequência dentre as $\mathrm{DMC}$ em diferentes países [5], e apresenta fraqueza acentuada nos músculos das cinturas escapular e pélvica [35]. Enquanto que na $\mathrm{MM}$ a fraqueza é distal, principalmente nos músculos das panturrilhas [7].

Diversos estudos foram realizados direcionados para a caracterização do acometimento e da evolução da doença, demonstrando alto grau de variabilidade no padrão de fraqueza, no entanto há escassez de pesquisas sobre biomarcadores funcionais.

Este trabalho com a exploração aprofundada do padrão de envolvimento muscular, da fraqueza e das habilidades motoras possibilita a geração de ferramenta confiável de acompanhamento evolutivo da doença para elaboração de pesquisas longitudinal e ensaios clínicos para melhora da prática clínica.

O objetivo deste estudo foi caracterizar a força e função muscular em observação transversal de prontuário de amostra de pacientes com disferlinopatia. Como objetivo secundário: investigar a correlação entre diferentes variáveis clínicas, considerando dois grupos de pacientes, cadeirantes e não cadeirantes.

\section{Métodos}

Estudo aprovado pelo Comitê de Ética em Pesquisa da Faculdade de Medicina da Universidade de São Paulo (protocolo 64577316.5.0000.0065).

Pesquisa transversal, descritiva, retrospectiva, com uso do banco de dados de doenças neuromusculares genéticas do Centro de Estudo sobre o Genoma Humano e Células Tronco (CEGH) do Departamento de Biologia - Instituto de Biociências da Universidade de São Paulo. Todos os pacientes acompanhados no Centro assinam termo de Consentimento Livre e Esclarecido. 
Coleta de dados e participantes

Grupo de 1340 pacientes com diversas doenças neuromusculares são acompanhados no setor de Fisioterapia Neurofuncional do CEGH. A amostra contou com 40 pacientes com diagnóstico fechado de disferlinopatia. O diagnóstico foi concluído pelo meio de exame de DNA pelo sequenciamento de nova geração (NGS) em $42,5 \%$ da amostra e por biópsia muscular em $57,5 \%$ dos casos.

As informações clínico-funcionais foram coletadas a partir do prontuário de avaliação utilizado no Setor de Fisioterapia. Os dados coletados foram: sexo, idade, início dos sintomas da doença, data do diagnóstico, idade da perda da marcha, presença de sinais clínicos específicos, função cardiorrespiratória, presença de comorbidades, força muscular, índice de força muscular, tempo de execução para deambular.

A MRC é mensurada por meio da escala Medical Research Council (MRC) [8]. Utiliza-se escala de 5 pontos, nos quais: grau zero (ausência de evidência de contração muscular), grau 1 (contração impotente que é sentida na palpação do músculo ou do tendão, porém sem movimento visível), grau 2 (o músculo é capaz de completar a amplitude de movimento no plano horizontal, sem ação da gravidade), grau 3 (o músculo pode manter a posição da prova contra a resistência da gravidade), grau 4 (o músculo pode manter a parte na posição da prova contra pressão moderada) e grau 5 (o músculo pode ser mantido na posição da prova contra forte pressão). Os grupos musculares utilizados: flexores da cervical, extensores da cervical, flexores do tronco, extensores do tronco, deltoides (porções anterior, média e posterior), bíceps braquial, tríceps braquial, flexores dos punhos, extensores dos punhos, iliopsoas, glúteo, adutores do quadril, isquiotibial, quadríceps femoral, gastrocnêmio e tibial anterior.

Coletou-se o índice do MRC [9] das regiões proximal dos membros superiores (MMSS), distal dos MMSS, proximal dos membros inferiores (MMII) e distal dos MMII, calculado por meio da fórmula: Índice MRC (\%) = soma da pontuação dos testes (bilateral) x 100 /número de músculos testados $\times 5$.

O tempo de execução para deambular coletado do prontuário foi realizado em corredor plano de 10 metros e utilizando-se cronômetro simples. A realização desta atividade fornece dados para comparações do próprio paciente e deste com indivíduos com outros diagnósticos como distrofia muscular de Duchenne (DMD) [10]. 
Escalas clínicas

Foram coletados dos prontuários os escores das escalas clínicas de Vignos modificada [11], que classifica o estadiamento da doença por meio do desempenho motor e a escala Egen Klassifikation (EK), validada para português [12], que quantifica o grau de limitação funcional nas atividades de vida diária em pacientes com DMD em fase crônica.

Coletou-se do prontuário informações da descrição qualitativa da realização de atividades motoras como levantar do chão, sentar e levantar da cadeira, ficar na ponta dos pés, ficar nos calcanhares, andar, subir e descer escadas, pular, correr, agachar-se e levantar-se. Estes dados foram utilizados para inferir as escalas de Escala de Avaliação Funcional para DMD/Functional Evaluation Scale (FES-DMD) e North Star Ambulatory Assessment adaptada (aNSAA), ambas ainda não validadas.

A FES-DMD é a escala brasileira, com boa concordância na repetibilidade e reprodutibilidade, composta por quatro domínios, são eles: sentar e levantar da cadeira, sentar e levantar do chão, subir e descer escadas e deambular. O domínio utilizado foi o deambular [10], que é composto de três fases e 14 itens. A primeira fase, "Apoio" com cinco itens e variação de pontos de 0 a 23, a segunda, "Balanço" com seis itens e pontuação entre 0 e 11 e a terceira, "Movimentos Compensatórios Gerais" com seis itens e variação de pontos de 0 a 13.

A North Star Ambulatory Assessment adaptada (aNSAA) foi elaborada para pacientes com disferlinopatia [2], a partir da necessidade de avaliação de aspectos específicos da doença como fraqueza proximal e fraqueza distal dos MMII. Tem como base a NSAA, escala validada para avaliação funcional de pacientes com DMD, com boa confiabilidade e boa a moderada responsividade. A versão original contém 17 itens que abordam a capacidade de permanecer em pé, deambular, levantar da cadeira, manter apoio unipodal, subir degraus, sentar, levantar do chão, levantar a cabeça, pular em apoio unipodal, saltar, ficar nos calcanhares e correr. As atividades são pontuadas entre 0 (necessidade de assistência), 1 (execução de forma independente com compensações) e 2 (execução normal) [13]. Para a aNSAA foram acrescidos 5 itens com pontuação máxima de 51 . Posteriormente os autores construiram a estratificação de gravidade do quadro clínico, no qual o quadro é severo para pontuações $\leq 5$ ou em cadeirantes, quadro moderado para pontuação entre 6 e 39 e quadro leve para pontuação entre 40 a 51 . 
Análise de dados

Análise estatística descritiva com cálculo do valor de média e desvio-padrão utilizados para descrever as características gerais da amostra. Testou-se a normalidade de cada variável por meio do teste de aderência Kolmogorov-Smirnov. Posteriormente, realizou-se o teste de correlação de Pearson com o objetivo de investigar possíveis relações entre as variáveis. A análise de variância foi utilizada para verificar a comparação entre a MRC nos seguimentos corporais. Em seguida, a análise de PostHoc de Tukey. A análise estatística dos dados foi realizada por meio da utilização do software Statistica v.13 e o nível de significância adotado foi de 0,05.

\section{Resultados}

Dados demográficos

Dos 1.340 prontuários de pacientes acompanhados no Setor de Fisioterapia do CEGH-USP, a DMC é a quarta doença mais frequente entre os diagnósticos fechados compreendendo 10,5\% dos casos. Entre as DMC, a DMC2B é a segunda mais frequente em prevalência com $25,5 \%$, atrás apenas da calpainopatia com $34 \%$.

Apenas três pacientes apresentaram início dos sintomas antes dos 15 anos. Presença de 6 irmandades (Tabela I).

Tabela I - Dados demográficos da amostra

\begin{tabular}{ll}
\hline Dados demográficos & \\
\hline Idade & Média $36,5 \pm 10,2$ anos \\
Idade mínima & 18 anos \\
Idade máxima & 62 anos \\
Sexo masculino & 21 pacientes $(52,5 \%)$ \\
Sexo feminino & 19 pacientes $(47,5 \%)$ \\
Idade início dos sintomas & Média $23,7 \pm 9$ anos \\
Idade mínima do início dos sintomas & 11 anos \\
Idade máxima do início dos sintomas & 54 anos \\
Tempo de doença & Média $12,8 \pm 7,1$ anos \\
Deambuladores & 30 pacientes $(75 \%)$ \\
Cadeirantes & 10 pacientes $(25 \%)$ \\
Tempo de marcha & Média $17,5 \pm 4,4$ anos \\
Idade de perda da marcha & Média $39,6 \pm 10$ anos \\
Idade mínima de perda de marcha & 27 anos \\
Idade máxima de perda da marcha & 55 anos \\
Presença de dores musculares & 23 pacientes $(57,5 \%)$ \\
Presença de fadiga muscular & 20 pacientes $(50 \%)$ \\
\hline
\end{tabular}


Características clínicas gerais

Encontrou-se presença de hipotonia muscular em 15\% da amostra. A atrofia muscular esteve presente em $82,5 \%$, sendo as regiões mais acometidas: panturrilhas e coxas. A pseudohipertrofia muscular foi observada em apenas três pacientes, dois pacientes, nos músculos das panturrilhas e um paciente, nos músculos bíceps braquiais e deltóides (Figura 1).

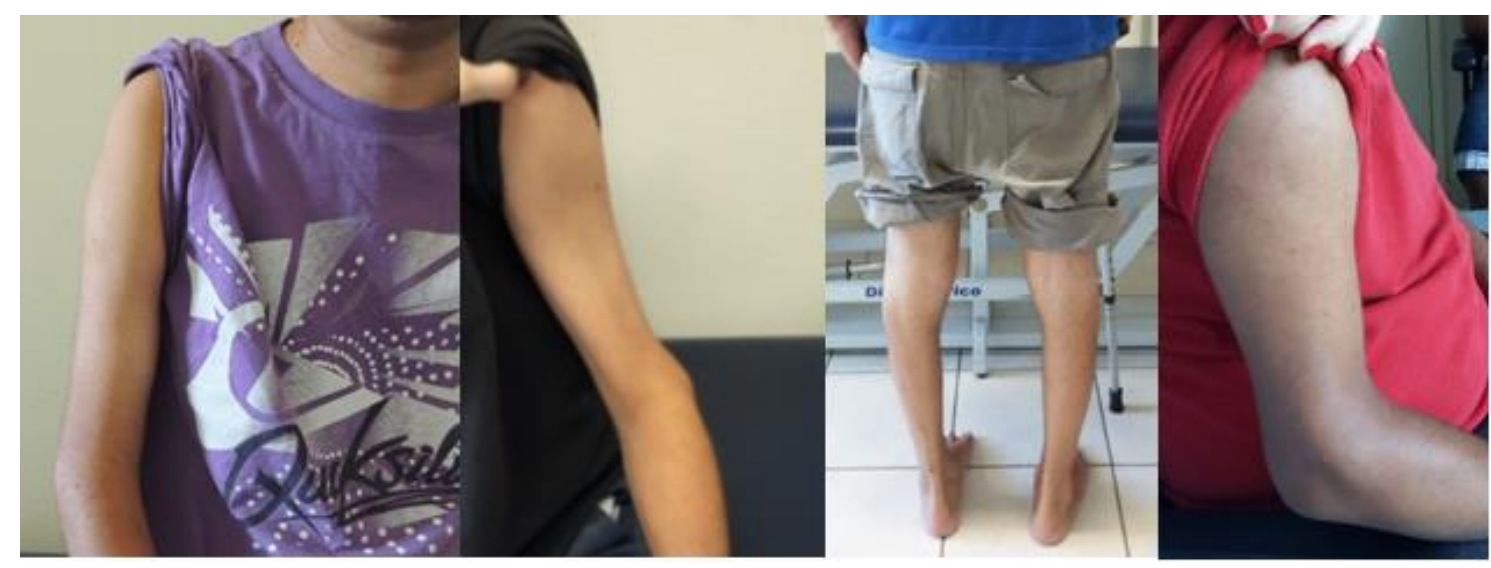

Figura 1 - Atrofia e pseudohipertofia muscular em 4 pacientes. Da esquerda para direita: atrofia de deltoide no sujeito 16; atrofia de bíceps braquial no sujeito 2; atrofia das panturrilhas no sujeito 36; pseudohipertrofia do deltoide com atrofia do bíceps braquial no sujeito 21

Força muscular

A MRC cervical estava preservada em $97,5 \%$ da amostra (MRC $\geq 3$ ). A fraqueza dos músculos abdominais e paravertebrais foi observada em $72,5 \%$ e $55 \%$, respectivamente.

A fraqueza dos MMSS foi mais acentuada nos músculos proximais, sendo o músculo deltoide anterior mais acometido com média de MRC 3,1 em 50\% dos casos, seguido do deltoide anterior (média de MRC 3,2) e do deltoide posterior (média de MRC $3,1)$. O bíceps e tríceps braquial tiveram média MRC de 3,7 e os flexores e extensores dos punhos média de MRC 4,2 e 4,3, respectivamente. Não se observou MRC 1 nos músculos dos MMSS. Nos MMII, a fraqueza esteve presente nos músculos proximais e distais, sendo o iliopsoas e o glúteo os músculos mais acometidos (média de MRC 2,5), seguido do isquiotibial (média de MRC 2,6), adutores do quadril e tibial anterior (média de MRC 2,7). O gastrocnêmio e o quadríceps femoral apresentaram média de MRC de 3,2 e 3, respectivamente. Observou-se MRC 1 em adutores do quadril, quadríceps, isquiotibial e tibial anterior. 
Nos MMII, em ambos os grupos (deambuladores e cadeirantes), verificou-se na articulação do joelho a preservação da força do compartimento anterior, ou seja, quadríceps femoral ( $p=0,003$ ); enquanto que na articulação do tornozelo, o músculo do compartimento posterior (gastrocnêmio) esteve mais preservado $(p=0,02)$. Não houve diferença entre a MRC da articulação do quadril (iliopsoas e glúteo).

Pelo índice de MRC verificou-se que no grupo deambuladores a força dos MMSS, tanto proximal quanto distal, está preservada, enquanto há fraqueza dos MMII de predomínio proximal. No grupo cadeirantes, há fraqueza da região proximal dos MMSS e intensa fraqueza dos MMII (proximal e distal, em semelhante proporção) (Figura 2).
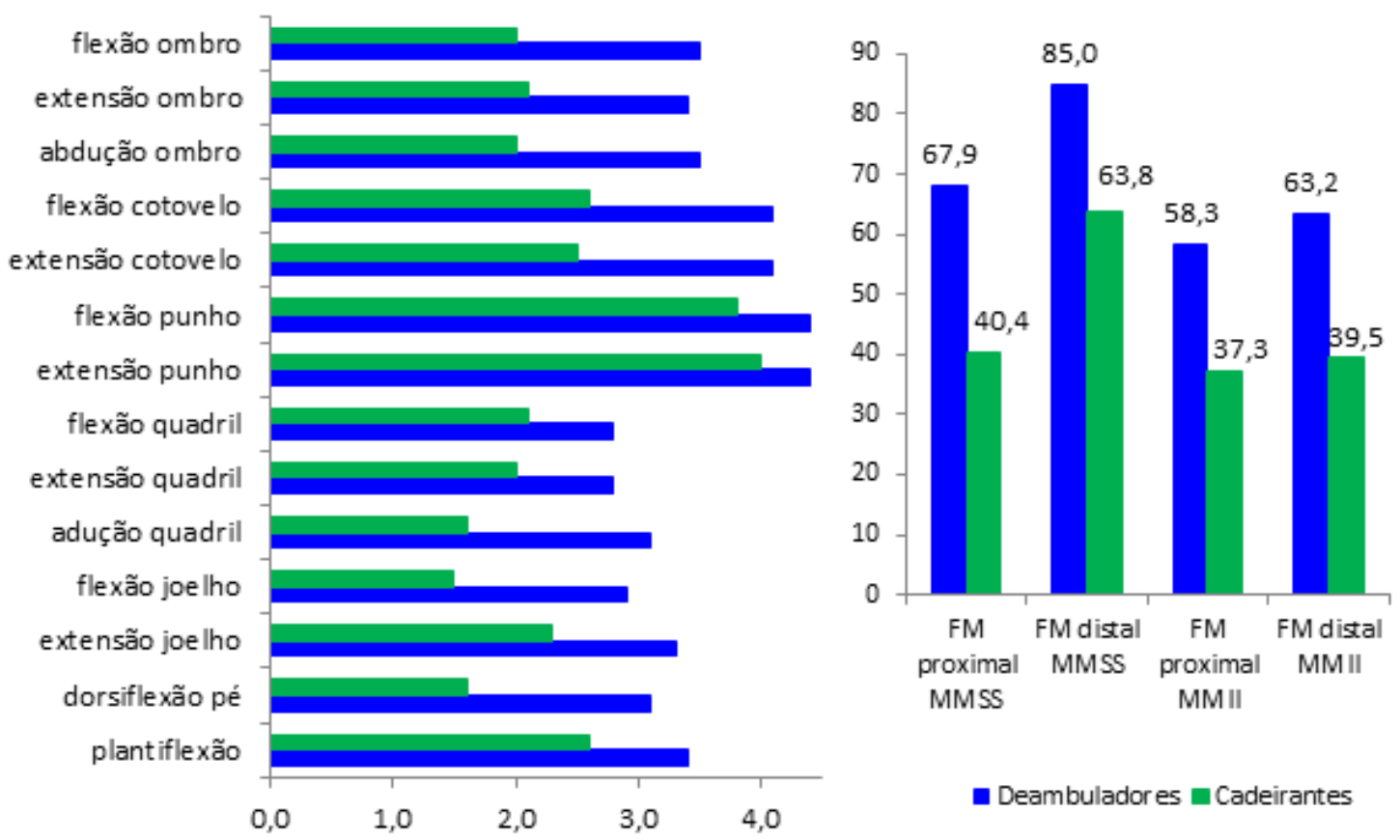

FM = força muscular; MRC = Medical Research Council; MMSS = membros superiores; MMII: membros inferiores

Figura 2 - Comparação da força muscular manual. À esquerda: média FM nos membros; à direita: média do índice de MRC nos membros

\section{Função cardiorrespiratória}

Os dados da função respiratória do teste de função pulmonar foram coletados do prontuário e encontrou-se que $42,5 \%$ da amostra possuía padrão restritivo em declínio, 35\% não tinha alteração e, portanto, apresentavam teste normal e $22,5 \%$ possuíam padrão restritivo leve.

As informações sobre as avaliações cardíacas foram retiradas das anotações do prontuário dos laudos médicos dos exames de eletrocardiograma, ecocardiograma e 
Holter. A ausência de alterações cardíacas foi encontrada em 27,5\% da amostra, enquanto que $22,5 \%$ possuíam alguma alteração cardíaca. Metade da amostra não possuía exames cardíacos. As alterações cardíacas encontradas no prontuário foram: alteração de relaxamento do miocárdio, arritmia cardíaca, cardiopatia leve, cardiopatia moderada, disfunção diastólica do ventrículo esquerdo, dilatação discreta do ventrículo esquerdo, distúrbio inespecífico de condução intraventricular, hipertrofia concêntrica do ventrículo esquerdo, infarto agudo do miocárdio, sobrecarga do ventrículo esquerdo e taquicardia sinusal.

A presença de comorbidades foi citada em $20 \%$ da amostra, sendo elas: dislipidemia, espodiloartrose, etilismo social, hipertensão arterial, hiperuricemia, hipotireoidismo e tabagismo.

\section{Correlações entre variáveis funcionais}

MRC e dados demográficos

Houve forte correlação entre idade e início dos sintomas $(r=0,73)$ e moderada correlação entre idade e tempo da doença $(r=0,49)$. A correlação da idade com a MRC foi fraca para todos os seguimentos corporais: proximal dos MMSS $(r=-0,34)$, distal dos MMSS $(r=-0,29)$, proximal dos MMII $(r=-0,18)$ e distal dos MMII $(r=-0,21)$.

MRC e escalas clínicas

A correlação da MRC e do escore da aNSAA foi forte com as regiões proximal dos MMSS e MMII ( $r=0,83,0,80$, respectivamente), moderada com distal dos MMSS ( $r$ $=0,69)$ e muito forte com distal dos MMII $(r=0,90)$ (Figura 3). 

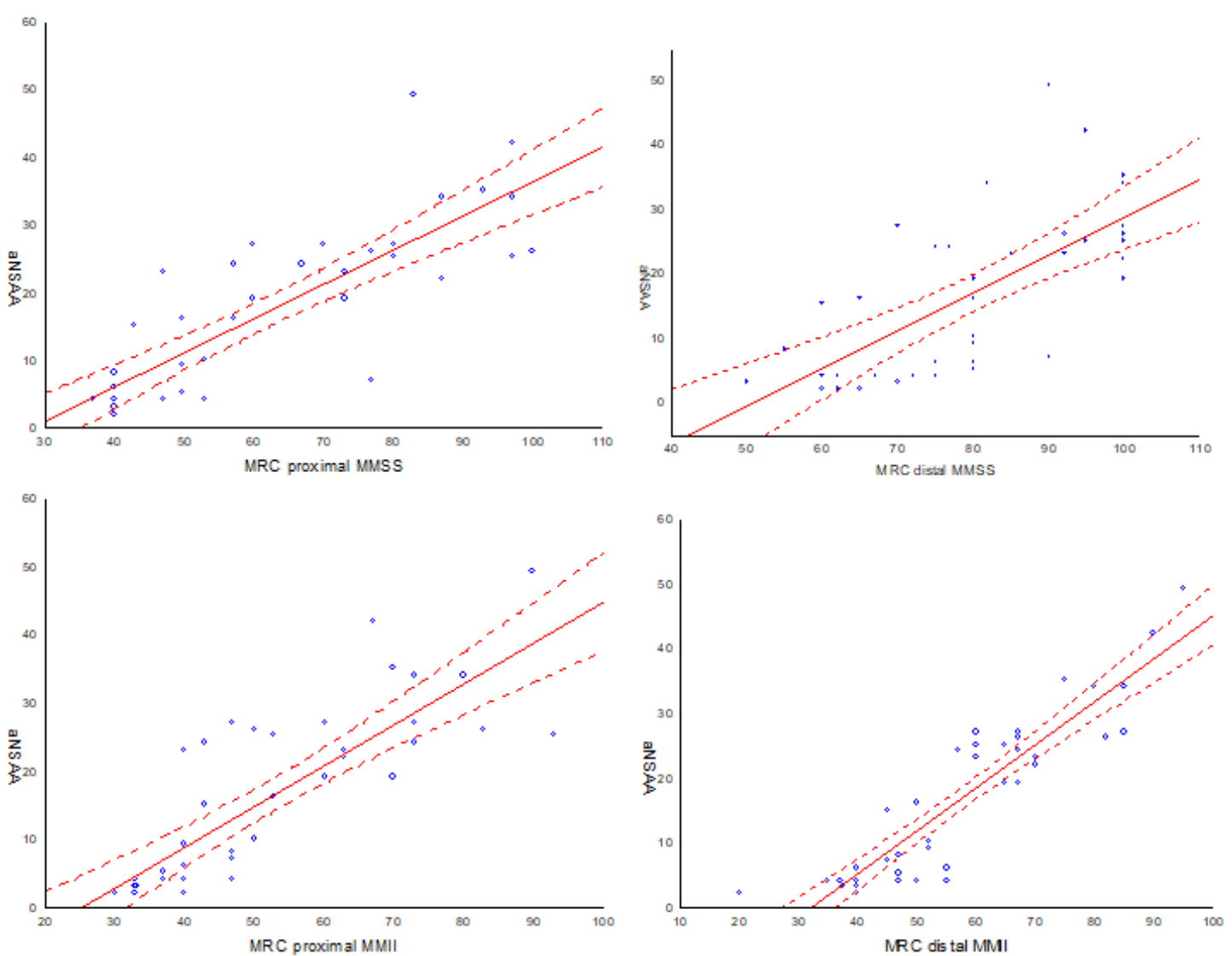

MRC = Medical Research Council; MMSS = membros superiores; MMII = membros inferiores; aNSAA = North Star Ambulatory Assessment adaptada

Figura 3 - Correlação entre as variáveis MRC e aNSAA. À esquerda no alto: MRC

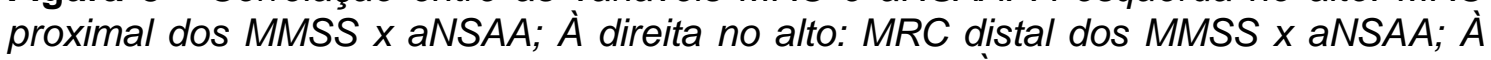
esquerda embaixo: MRC proximal dos MMII x aNSAA; $\dot{A}$ direita embaixo: MRC distal dos MMII x aNSAA

A correlação entre a MRC e o tempo de execução para deambular foi forte para as regiões proximal dos MMSS, proximal dos MMII e distal dos MMII $(r=-0,77,-0,74$ e $-0,80$, respectivamente). A correlação foi moderada com a região distal dos MMSS ( $r=-$ $0,68)$.

Os escores de Vignos da aNSAA tiveram forte correlação $(r=-0,86)$. O tempo para execução para deambular obteve correlação muito forte com escore da Vignos ( $r$ $=0,92)$ e forte com escore das escalas de FES-DMD e aNSAA $(r=0,87$ e $r=-0,84$, respectivamente).

A estratificação de gravidade da doença por meio do escore da aNSAA verificou 2 pacientes na categoria leve, 26 pacientes na categoria moderada e 12 pacientes na categoria grave.

A análise demonstrou que a proporção de pacientes mais graves esteve nas categorias com maior tempo de doença. A taxa de progressão da doença foi variável, com $60 \%$ dos pacientes moderadamente ou gravemente afetados com $>12$ anos de 
doença, enquanto que a proporção de indivíduos gravemente afetados foi de $100 \%$ após 24 anos de tempo de doença (Figura 4).

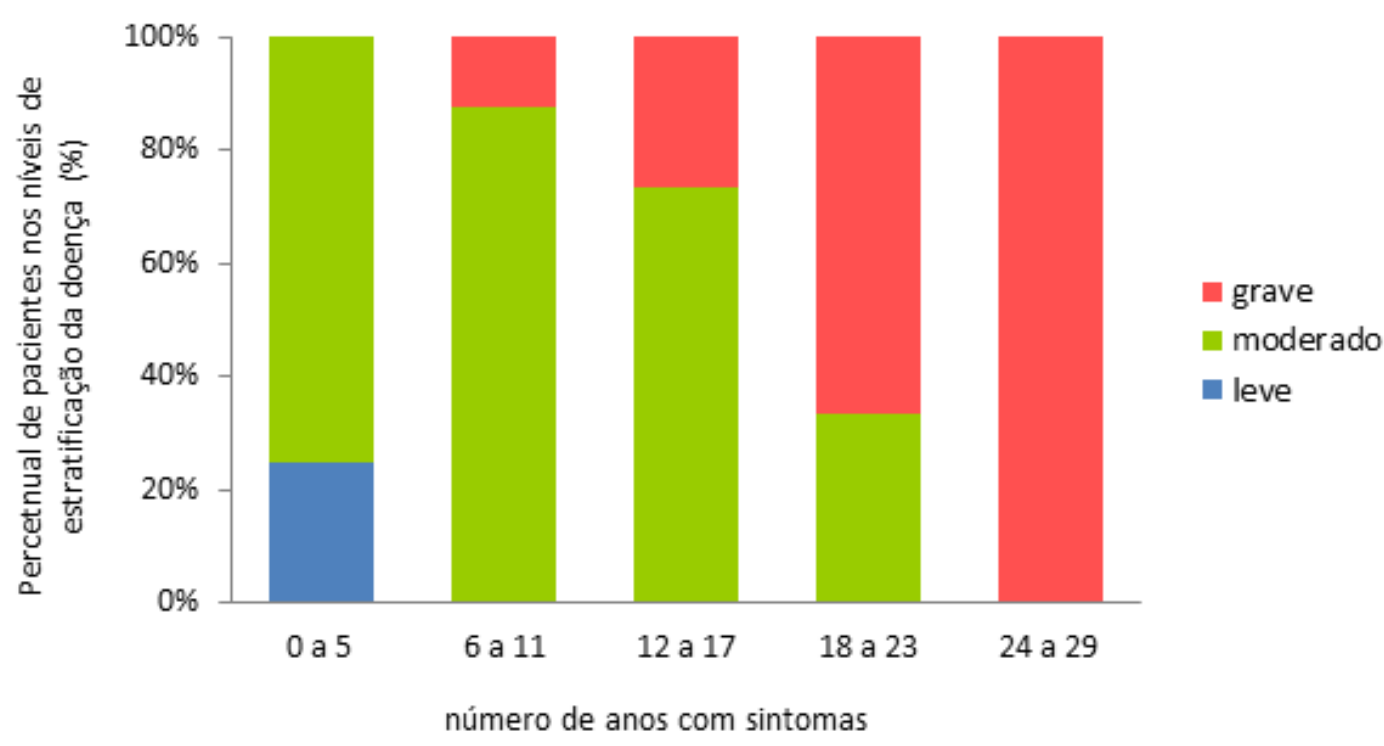

aNSAA $=$ North Star Ambulatory Assessment adaptada

Figura 4 - Porcentagem de pacientes dentro de cada categoria de gravidade por meio da estratificação do escore da escala de aNSAA

Padrão de MRC nos segmentos corporais

A análise de variância demonstrou que houve interação membro versus região versus grupo (cadeira ou deambulador), apresentando $F(1,38)=3,79 ; E S=0,090 ; p<$ 0,001 (Figura 5).

A análise Post-Hoc de Tukey demonstrou que no grupo cadeirante, houve diferença entre a MRC proximal e distal dos MMSS $(p<0,001)$, e entre a MRC distal dos MMSS e a proximal e distal dos MMII $(p<0,001)$. Não houve diferença entre a MRC proximal dos MMSS e proximal e distal dos MMII $(p=0,960$ e $p=0,999$, respectivamente) e, entre a MRC proximal e distal dos MMII $(p=0,995)$.

No grupo deambuladores, houve diferença significante entre a MRC proximal e distal dos MMSS $(p<0,001)$, entre a MRC proximal dos MMSS e MMII $(p<0,001)$, entre MRC distal dos MMSS e proximal e distal dos MMII $(p<0,001)$. Não houve diferença entre as MRC proximal e distal dos MMII $(p=0,084)$.

A análise de interação entre cadeirantes e deambuladores demonstrou que não houve diferença significante entre MRC proximal dos MMSS dos grupos $(p=0,003)$, MRC distal dos MMSS dos grupos ( $p=0,0434$ ), MRC proximal dos MMII dos grupos ( $p$ $=0,048)$ e MRC distal dos MMII dos grupos $(p=0,015)$. 
Em suma, encontrou-se diferença entre as regiões proximal e distal, sendo esta diferença mais evidente em MMSS do que em MMII; ou seja, a MRC geral dos MMII é bastante diminuída; enquanto a MRC distal de membros superiores é relativamente preservada.

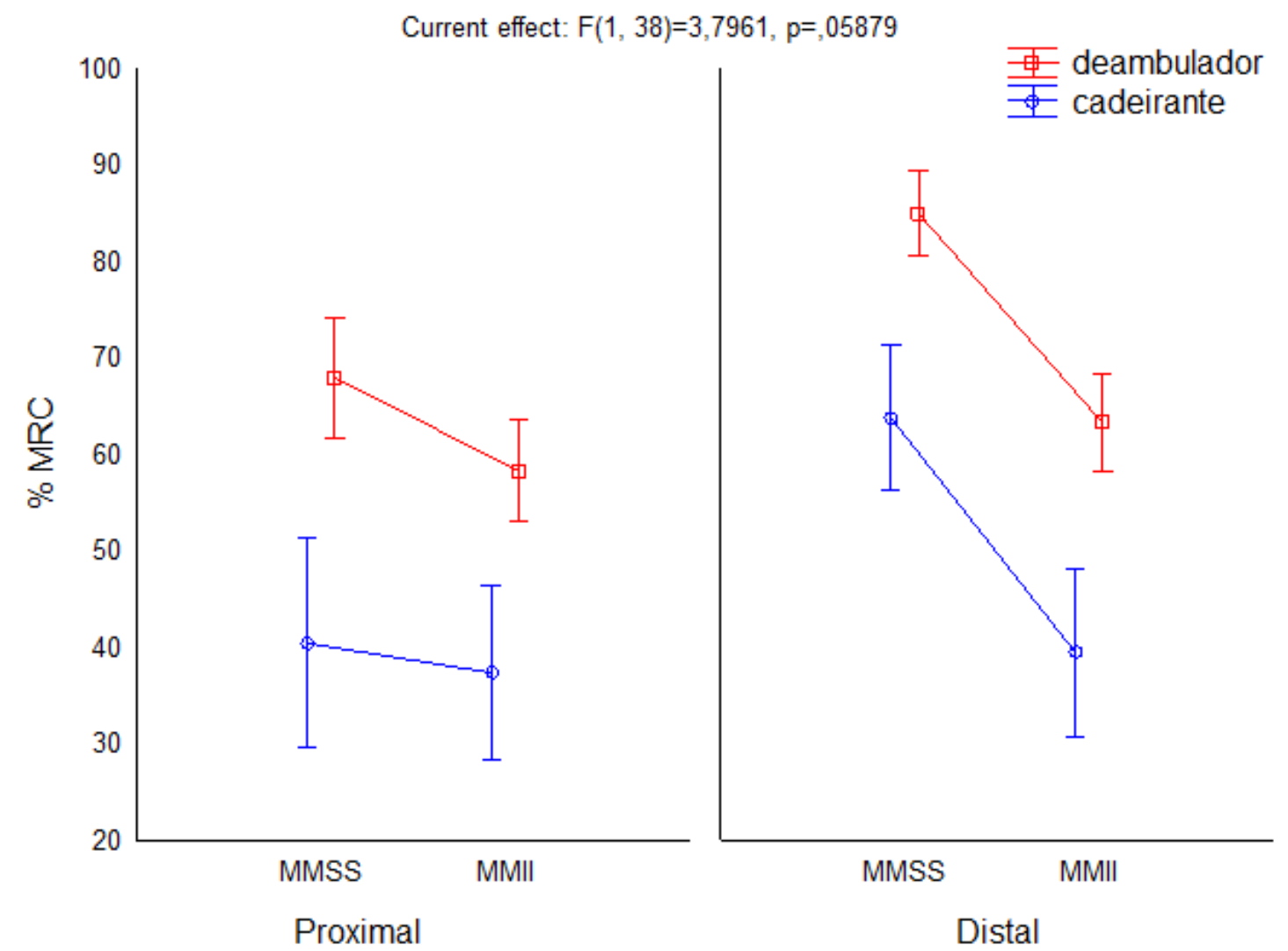

MRC $=$ Medical Research Council; MMSS $=$ membros superiores $;$ MMII $=$ membros inferiores

Figura 5 - Interação entre o índice de MRC nos seguimentos corporais - MMSS, MMII, região proximal e região distal nos grupos deambuladores e cadeirantes

\section{Discussão}

Esta pesquisa apresentou o primeiro estudo clínico-funcional de amostra brasileira de 40 pacientes com disferlinopatia, a quarta DMC mais frequente no CEGH/USP com prevalência de 25,5\%, condizente com a prevalência encontrada na literatura [14]. Estudo brasileiro de 2001 [15] identificou prevalência mais baixa (14\%), entretanto, naquele período o diagnóstico não era realizado pelo NGS, exame este que possibilitou a expansão dos diagnósticos de diversos tipos de distrofias, especialmente as DMC.

Média de idade da amostra de 36,5 anos (variação de 18 a 62), mais velha quando comparada a outras pesquisas (média variando de 20 a 27,9 anos) [14,16]. Entretanto, mais jovem quando comparada a amostra japonesa (média 50,3 anos, 
variação de 22 a 75) [1]. Ligeira prevalência do sexo masculino $(52,5 \%)$ [6,17], porém diferentemente da prevalência feminina em países orientais e na população mexicana $[18,19]$. Início dos sintomas, variação de idade e presença de sintomas semelhante à literatura [20-22].

O tempo de doença não é homogêneo na literatura. Neste estudo foi encontrado tempo médio de 12,8 anos (variação de um a 29 anos), menor que o encontrado pela pesquisa japonesa (23,7 anos) [1], e maior que a indiana (6,4 anos) [14]. A discrepância pode estar relacionada a fatores como acesso ao diagnóstico precoce, acesso à saude, conhecimento da doença por parte dos profissionais de saúde, qualidade de vida e expectativa de vida. Shin et al. (2015) [19] relatam média entre o início dos sintomas até o diagnóstico de 7,5 anos com intervalo de um a 30 anos.

Presença de grande maioria de deambuladores (75\%) condizente com a literatura que considera as disferlinopatias mais leve quando comparadas a calpainopatia e a sarcoglicanopatia [16], porém menos leve quando comparada a DMC2L, distrofia causada pela ausência de anoctamia 5 [21]. A média de idade da perda da marcha (39,6 anos) também acompanha a literatura [23], e a ausência de alterações cardíacas importantes e o padrão respiratório restritivo em declínio também foram descritos [1].

A atrofia muscular das panturrilhas encontrada nesta pesquisa também foi largamente relatada nos pacientes com disferlinopatia [21,22]. Entretanto, há pouco relato sobre a presença de atrofia do músculo bíceps braquial associada à preservação do músculo deltoide, resultando em pseudohipertrofia [16].

Encontramos nos MMSS predomínio de fraqueza proximal, em que a seletividade do envolvimento muscular foi típica, tal como descrito nas DMC, primariamente afetado o músculo deltoide, e secundariamente o bíceps e o tríceps braquial $[1,14]$ e, com as mãos relativamente preservadas [19,20]. Nos MMII, verificouse fraqueza proximal e distal [14,16,21-23], principalmente no grupo de cadeirantes. $O$ escore MRC 1 foi observado nos músculos adutores do quadril, quadríceps, isquiotibial e tibial anterior, compatível com casos mais avançados [1].

Quanto ao padrão de acometimento, observou-se preservação de força do grupo muscular anterior na articulação do joelho (quadríceps femoral), porém fraqueza de tibial anterior quando comparado ao gastrocnêmio. A fraqueza de tibial anterior também foi relatada na população alemã [21]. Não houve diferença entre a MRC de iliopsoas e glúteo, diferentemente do relatado pela literatura com flexores do quadril mais fortes que extensores [2].

O índice de MRC demonstra que a fraqueza proximal dos MMSS está presente nos cadeirantes e a fraqueza distal dos MMIl é presente em ambos os grupos, porém 
mais intensamente nos cadeirantes. A partir destes dados, pode ser sugerida a proporção da perda da força, com queda mais acentuada em MMII e o aumento da fraqueza proximal dos MMSS relacionado com doença mais grave ou quadro mais avançado, assim como corte inglesa ( $\mathrm{N}=36)$, na qual, a força dos MMSS diminuiu para $76 \%$, enquanto a queda nos MMII foi para 49\% [22] e, coorte multicêntrica ( $N=193)$ com perda de força muscular mais evidente na região distal dos MMII (71\% da amostra) [2].

Apesar de descritas na literatura, as diferenças entre as doenças conhecidas como disferlinopatias, no qual a DMC2B é caracterizada por fraqueza proximal e a MM é descrita por fraqueza distal com envolvimento precoce dos músculos do compartimento anterior da perna [24], verificam-se relatos com discrepâncias nos grupos musculares mais acometidos dos MMII [2,22]. Neste estudo foi observada equiparação das fraquezas proximais e distais dos MMII, o que tornou difícil a distinção do envolvimento inicial. Este achado também encontrado na literatura reflete a progressão da doença com sobreposição de fraqueza proximal e distal, corroborando que, embora as disferlinopatias possam apresentar inicialmente fenótipos distintos, elas se sobrepõem em estágios mais avançados [2,22].

Este estudo apresentou dados robustos relativos a habilidades motoras dos pacientes com disferlinopatia, com correlação da MRC, principalmente dos MMII, com o tempo de execução para deambular e as escalas clínicas de Vignos, EK, FES-DMD e aNSAA, o que reforça a relevância destas variáveis como biomarcadores da evolução das disferlinopatias. Em sua grande maioria, os estudos utilizam a escala de Vignos modificada ou, a exemplificação da gravidade do quadro por meio da descrição da incapacidade de levar as mãos à boca ou o confinamento no leito $[1,19,20]$.

A estratificação da escala aNSAA demonstrou que $65 \%$ dos pacientes deste estudo estão na categoria moderada, $30 \%$ na grave e, $5 \%$ na leve; enquanto que a coorte multicêntrica [2] identificou 49\%, 32\% e 19\%, respectivamente. Nossa pesquisa apresentou idade média e tempo de doença ligeiramente mais baixos (36,5 anos versus 40 anos e, 12,8 anos versus 17 anos, respectivamente). A coorte apresentou casos mais brandos com indivíduos em categoria leve de gravidade em todos as faixas de idade, enquanto que neste trabalho a categoria leve está restrita a faixa de até 5 anos de doença.

Nos pacientes com disferlinopatia a fraqueza de músculos proximais e distais dos MMII pode comprometer seriamente a capacidade de deambulação, visto que, em estudos com diferentes doenças neuromusculares, pacientes com fraqueza distal dos MMII apresentam movimentos de tronco, joelhos e tornozelos instáveis e aumentados durante os desequilíbrios direitos e para trás, em contraste, aqueles com fraqueza 
proximal, com pior resposta de equilíbrio apenas nas perturbações posteriores [25]. A fraqueza do compartimento posterior (isquiotibial, gastrocnêmio e sóleo) pode ainda inferir padrão distinto de alteração na marcha com perda do choque do calcâneo e da propulsão da marcha e ausência de extensão do joelho durante as fases de apoio e balanço [20].

Os estudos enfatizam o esforço e a necessidade do diagnóstico para classificar os pacientes com disferlinopatias, considerando-se a combinação de dados clínicos, imuno-histoquímica e dados moleculares genéticos. Chama a atenção o relato de proporção significativa de pacientes classificados como DMC (27,9\% a 41,3\%) [26] e evidencia-se variação das frequências de subtipos confirmados em populações diferentes, que pode ser explicada por testes bioquímicos e imunológicos menos abrangente nas biópsias musculares e/ou testes genéticos. Paralelamente, reitera que a grande variabilidade do padrão de fraqueza e da taxa de progressão corroborando a necessidade de estudos longitudinais [2] e da determinação de biomarcadores [27,28] para melhor elucidar a trajetória dos fenótipos e apontar para desenvolvimento de estudos clínicos de intervenções mais eficazes e seguros [2].

\section{Conclusão}

Este estudo identificou o padrão de fraqueza de amostra brasileira de pacientes com disferlinopatia. A partir do conhecimento gerado por estes dados, pode-se fazer interface com a pesquisa translacional, dando suporte para escolha do biomarcador de acompanhamento da doença e gerando informações relevantes para tomada de decisões clínicas. A intervenção fisioterapêutica deve ser direcionada para o treinamento dos movimentos do joelho no auxílio as respostas de equilíbrio, o treino das estratégias de equilíbrio compensatórias do tornozelo e de adaptação de atividades de vida diária que dependam da elevação dos MMSS acima da cabeça. Estudos futuros devem focar na determinação do efeito dos exercícios na resistência muscular, no efeito do uso de órteses funcionais na estabilidade e no equilíbrio corporal e na correlação do uso de órtese com o prolongamento do tempo de deambulação.

As limitações encontradas referem-se à escassez de informações sobre a evolução da fraqueza muscular que permitisse categorizar os pacientes nos diferentes tipos de disferlinopatias, à necessidade de validação da escala funcional específica para disferlinopatia e à correlação com outra escala funcional validada e de maior utilização nas miopatias e distrofias. A ampliação da avaliação com uso de escalas validadas, bem como a ampliação da pesquisa com estudos longitudinais, é interessante e necessária 
para auxiliar na elucidação do desenvolvimento da doença e no desenvolvimento de estudos clínicos fisioterapêuticos.

\section{Agradecimentos}

Dra. Mayana Zatz por ceder os dados para este estudo; Equipe Centro de Estudos do Genoma Humano e Células Tronco pela consultoria técnica; A North Star Ambulatory Assessment adaptada (aNSAA), pela cessão de avaliação para este estudo.

\section{Referências}

1. Takahashi T, Aoki M, Suzuki N, Tateyama M, Yaginuma C, Sato H, et al. Clinical features and a mutation with late onset of limb girdle muscular dystrophy 2B. J Neurol Neurosur Ps 2013;84(4):433-40. doi: 10.1136/jnnp-2011-301339

2. Harris E, Bladen CL, Mayhew A, James M, Bettinson K, Moore U, et al. The clinical outcome study for dysferlinopathy: an international multicenter study. Neurol Genet 2016;2(4):e89. doi: 10.1212/NXG.0000000000000089

3. Manzur AY, Muntoni, F. Diagnosis and new treatments in muscular dystrophies. J NeurolNeurosur Ps 2009;80(7):706-14. doi: 10.1136/jnnp.2008.158329

4. Eulate FG, Querin G, Moore U, Behin A, Masingue M, Bassez G. Deep phenotyping of an international series of patients with late-onset dysferlinopathy. Eur $\mathrm{J}$ Neurol 2021;28(6):2092-102. doi: 10.1111/ene.14821

5. Nigro V, Savarese M. Genetic basis of limb-girdle muscular dystrophies: the 2014. Acta Myol [Internet]. 2014 [cited 2021 Dec 4];33(1):1-12. Available from: https://www.ncbi.nlm.nih.gov/pmc/articles/PMC4021627/

6. Diniz G, Eryaşar $G$, Türe $S$, Akçay $A$, Ortaç $R$, Tekgül $H$, et al. A regional panorama of dysferlinopathies. Turk Patoloji Derg 2012,28:259-65. doi: 10.5146/tjpath.2012.01133

7. Wicklund MP, Kissel JT. The limb-girdle muscular dystrophies. Neurol Clin 2014;32(3):729-49. doi: 10.1016/j.ncl.2014.04.005

8. Medical Research Council. Aids to the examination of the peripheral nervous system. Memorandum No. 45 (superseding War Memorandum No. 7) [Internet]. London: Her Majesty's Stationery Office. 63p.1976. [cited 2021 Dec 4]. Available from: https://www.mrc.ac.uk/research/facilities-and-resources-for-researchers/mrcscales/mrc-muscle-scale

9. Scott OM, Hyde SA, Goddard C, Dubowitz V. Quantitation of muscle function in children: a prospective study in Duchenne muscular dystrophy. Muscle Nerve 1982;5(4):291-301. doi: 10.1002/mus.880050405

10. Carvalho EV, Caromano FA. Criação e análise de confiabilidade de escala de avaliação funcional da marcha para crianças com distrofia muscular de Duchenne. [Dissertation] [Internet]. São Paulo: Universidade de São Paulo; 2013. Available from: http://www.teses.usp.br/teses/disponiveis/5/5170/tde-10122013-121610/pt-br.php

11. Gardner-Medwin D, Walton JN. Disorders of voluntary muscle. Edinburg: Churchill Livingstone; 1974; p.517-60. 
12. Martinez JA, Brunherotti MA, de Assis MR, Sobreira CF. Validation of the EK functional motor scale in the Portuguese language. Rev Assoc Med Bras (1992) 2006;52(5):34751. doi: $10.1590 / S 0104-42302006000500024$

13. Scott E, Eagle M, Maythew A, Freeman J, Main M, Sheehan J, et al. North Star Clinical Network for Paediatric Neuromuscular Disease. Development of a functional assessment scale for ambulatory boys with Duchenne muscular dystrophy. Physiother Res Int 2012;17(2):101-9. doi: 10.1002/pri.520

14. Nalini A, Gayathri N. Dysferlinopathy: a clinical and histopathological study of 28 patients from India. Neurol India 2008;56(3):379-85; discussion 386-7. doi: 10.4103/0028-3886.40964

15. Vainzof M, Anderson LV, McNally EM, Davis DB, Faulkner G, Valle G, et al. Dysferlin protein analysis in limb-girdle muscular dystrophies. J Mol Neurosci 2001;17(1):71-80. doi: 10.1385/JMN:17:1:71

16. Rosales XQ, Gastier-Foster JM, Lewis S, Vinod M, Thrush DL, Astbury C, et al. Novel 21. doi: $10.1002 /$ mus. 21650

17. Zhao Z, Hu J, Sakiyama Y, Okamoto Y, Higuchi I, Li N, et al. DYSF mutation analysis in a group of Chinese patients with dysferlinopathy. Clin Neurol Neurosurg 2013;115(8):1234-7. doi: 10.1016/j.clineuro.2012.11.010

18. Gómez-Díaz B, Rosas-Vargas H, Roque-Ramírez B, Meza-Espinoza P, RuanoCalderón LA, Fernández-Valverde $\mathrm{F}$, et al. Immunodetection analysis of muscular dystrophies in Mexico. Muscle Nerve 2012;45:338-45. doi: 10.1002/mus.22314

19. Shin HY, Jang H, Han JH, Park HJ, Lee JH, Kim SW, et al. Targeted next-generation sequencing for the genetic diagnosis of Dysferlinopathy. Neuromuscular Disord 2015;25(6):502-10. doi: 10.1016/j.nmd.2015.03.006

20. Mahjneh I, Marconi G, Bushby K, Anderson LVB, Tolvanen-Mahjneh H, Somer H. Dysferlinopathy (LGMD2B): a 23-year follow-up study of 10 patients homozygous for the same frameshifting dysferlin mutations. Neuromuscular Disord 2001;11(1):20-6. doi: 10.1016/S0960-8966(00)00157-7

21. Linssen WH, Voogt WG, Krahn M, Bernard R, Levy N, Wokke JH, et al. Long-term follow-up study on patients with Miyoshi phenotype of distal muscular dystrophy. Eur J Neurol 2013;20(6):968-74. doi: 10.1111/ene.12129

22. Klinge L, Aboumousa A, Eagle M, Hudson J, Sarkozy A, Vita G, et al. New aspects on patients affected by dysferlin deficient muscular dystrophy. J Neurol Neurosur Ps 2010;81(9):946-53. doi: 10.1136/jnnp.2009.178038

23. Nguyen K, Bassez G, Krahn M, Bernard R, Laforêt P, Labelle V, et al. Phenotypic study in 40 patients with dysferlin gene mutations: high frequency of atypical phenotypes. Arch Neurol 2007;64(8):1176-82. doi: 10.1001/archneur.64.8.1176

24. Illa I, Serrano-Munuera C, Gallardo E, Lasa A, Rojas-García R, Palmer J, et al. Distal anterior compartment myopathy: a dysferlin mutation causing a new muscular 
dystrophy phenotype. Ann Neurol 2001;49(1):130-4.

https://pubmed.ncbi.nlm.nih.gov/11198284/

25. Horlings CGC, Küng UM, van Engelen BGM, Voermans NC, Hengstman GJ, van Der Kooi AJ, et al. Balance control in patients with distal versus proximal muscle weakness. Neuroscience 2009;164(4):1876-86. doi: 10.1016/j.neuroscience.2009.09.063

26. Guglieri M, Magri F, D’Angelo MG, Prelle A, Morandi L, Rodolico C, et al. Clinical, molecular, and protein correlations in a large sample of genetically diagnosed Italian limb girdle muscular dystrophy patients. Hum Mutat 2008;29:258-66. doi:

10.1002/humu.20642

27. Barthélémy F, Wein N, Krahn M, Lévy N, Bartoli M. Translational research and therapeutic perspectives in dysferlinopathies. Mol Med 2011;17(9-10):875-82. doi: 10.2119/molmed.2011.00084

28. Mahmood OA, Jiang XM. Limb-girdle muscular dystrophies: Where next after six decades from the first proposal (Review). Mol Med Rep 2014;9(5):1515-32. doi: $10.3892 / \mathrm{mmr} .2014 .2048$

Este artigo de acesso aberto é distribuído nos termos da Licença de Atribuição Creative Commons (CC BY 4.0), que permite o uso irrestrito, distribuição e reprodução em qualquer meio, desde que o trabalho original seja devidamente citado. 